\title{
Intelligent Vehicle Theft Control using Embedded System
}

\author{
Saloni Shah \\ Student, Dept. of Computer \\ Engineering, D. J.Sanghvi \\ College of Engineering, \\ Mumbai
}

\author{
Siddhi Patel \\ Student, Dept. of Computer \\ Engineering, D. J.Sanghvi \\ College of Engineering, \\ Mumbai
}

\author{
Prof. Harish Narula \\ Assistant Professor, Dept. of \\ Computer Engineering, D. \\ J.Sanghvi College of \\ Engineering, Mumbai
}

\begin{abstract}
In today's world almost every common man owns a vehicle. Theft is a common issue which everyone faces in parking and sometimes driving near insecure places. Vehicle security and accident prevention is more challenging. So in order to bring a solution for this problem the proposed system can be implemented. Vehicle security enhancement and accident prevention system can be developed through tracking and locking, fuel theft, accident detection and prevention, driver fatigue, pollution control and speed limiting with efficient vehicle management system. The need for this is to provide security to the vehicles by engine locking system which prevents the vehicle from unauthorized access. It can also prove beneficial to find out the exact location of the accident. This proposed work is an attempt to design such a system using global positioning system (GPS) to determine the precise location of a object, person or other asset to which it is attached and to transmit this information using GSM modem.
\end{abstract}

\section{Keywords}

Vehicle tracking and locking, microcontroller, global positioning system (GPS), GSM

\section{INTRODUCTION}

With the help of evolving technologies, many different security systems have been developed for detection of car thefts and tracking the location of the cars. Despite the development of these security systems, according to National crime information center the loses are in billion because of stolen cars. The main drawbacks of failures of these systems in securing the cars was that they do not assist owners in recovering a stolen car, they do not let owner communicate with its car online even if the owner is certain that his car has been stolen, also the user cannot find out the current status of his car when his car is being used by some other third party.

The proposed security system in this paper is an integration of existing technology GPS that is currently used in location tracking and modern communication technology SMS. SMS is the easiest way that is used widely for communication purposes because they are inexpensive, convenient and messages can be transmitted and received with high reliability. This combination of GPS and GSM technologies will provide effective, real time vehicle location, and reporting of the status to the owners. It will let owners by sending an SMS to owners about where the vehicle is presently, where was it and for how long it was present there using GPS.

In the proposed system, whenever anyone tries to access the car that is open the door and sit on the driving seat, the owner of the car will be sent a message through the GSM model. If the owner feels that his car is used by an illegitimate user then he will send a message to GSM module to switch off the car. In response to this message the speed of the car engine will slow down gradually until it gets turned off. Once it gets turned off, the doors get locked. Thus, the thief cannot escape. This is how theft control will be implemented.

\section{SURVEY ON RELATED WORK}

S.Sonica et al's paper[1] introduces the concept of ITS. Intelligent Transport System (ITS) is used to identify the accident, and the location of the accident is immediately sent to the server. The server then finds a nearby hospital and emergency vehicle to be sent to the accident zone. However, newer systems with newer techniques were later developed.

Kai-Tai Song and Chih-Chieh Yang[2] have designed and built a real-time visual tracking system for the purpose of vehicle safety. A vehicle-tracking algorithm, automatically detect and track several moving objects, like cars and motorcycles, ahead of the tracking vehicle. Joint with the concept of focus of expansion (FOE) and view analysis, the built system can segment features of moving objects from moving background and offer a word of warning on real-time if a collision is likely to occur. The proposed algorithm uses a CMOS image sensor and NMOS embedded processor architecture. The constructed stand-alone visual tracking system is validated in real road tests. The results provided information of collision warning in urban artery with speed about $60 \mathrm{~km} /$ hour both during the day and the night.

In [3], An Advanced Driving Assistance System (ADAS) with haptic, visual, and audio feedback includes safety feature for collision avoidance. It is implemented by using motion planning Automation of a Neighborhood Electric Vehicle (NEV). The system provided vehicle cabin safety based on embedded system by modifying the existing modules. In this method, the IR Sensor is used to detect the obstacle in front of the vehicle. The vehicle could not move ahead if any obstacle is detected. This is helpful in avoiding accidents due to collision of vehicles with any static obstacles.

Later, a new method of using cloud for theft detection was discussed by Albert et al in their paper [4]. The proposed tracking system was based on cloud computing infrastructure. The sensors are used to monitor the fuel level, driver conditions, and speed of vehicle. All the data is transferred to the cloud server using a GSM enabled device. All the vehicles are equipped with GPS antenna to locate the place. To avoid the drunk and drive, the alcohol sensor is installed to monitor the driver status. This proposed technology has served its best application to avoid accidents on highways. 


\subsection{Proposed System}

The process starts with various components sensing the parameter from various parts of the vehicle. The information collected from these devices is then sent to the ADC and then ARM 7 microcontroller[7] will process the data. Send the data over GPS and GSM which will then forward the message to the owner of the vehicle.

As shown in Fig. 1, when the car starts running, the owner receives an SMS indicating the same. If this is an illegal activity i.e. if any intruders try to run the car, the owner can send an SMS to switch off the car. Then, the system will check the mobile number in the received message, to confirm that the number is authorized to access the security system. If the phone number is legal, the system will turn off the car. Engine motor speed is gradually decreased until the vehicle gets turned off. After the vehicle gets turned off, all the doors are locked. To open the door or restart the engine, an authorized person needs to enter the password by sending an SMS. In this method, tracking of vehicle location becomes easy with the help of GPS technology, and the door-lock feature ensures that the thief cannot get away from the car.

If the owner needs to track the vehicle, he/she has to send SMS that contains a special code, after which he/she will receive an SMS containing the GPS coordinates of the car. The SMS updates its content every predetermined period of time. Also, the car owner can connect another GSM modem with a laptop to track the vehicle immediately using Google Earth. The implemented tracking and security system can be used to monitor various parameters related to safety antitheft, emergency services and engine stall.

Fuel level can be monitored all the time so as to prevent fuel theft. A threshold value would be set to indicate a minimum level of fuel required in the vehicle. If the fuel level drops below this threshold value, an SMS is generated and sent to the owner, indicating about the situation. Vehicle security will be enhanced by ignition control system. Obstacle detection system stops the vehicle when an obstacle is detected using IR sensor and pollution detection helps in controlling pollution from vehicle using CO sensor. GSM and GPS are used for tracking the location of vehicle and for providing SHORT MESSAGE SERVICE (SMS) material.

\subsection{Block Diagram}

The Block diagram of Vehicle tracking and locking system based on GSM and GPS technology is shown in the Figure 1. It consists the power supply section, keyboard, GSM, GPS, ARM7 microcontroller, MAX232driver, relay driver, IR Transmitter, IR receiver, LCD, Accident Sensor, Fuel Sensor, Lane detection Sensor, Ultrasonic obstacle sensor, Vehicle Parking Motors, Emergency Button and door locker. The GSM board has a valid SIM card with a sufficient recharge amount to make outgoing calls. The circuits powered by $+5 \mathrm{v}$ Dc.

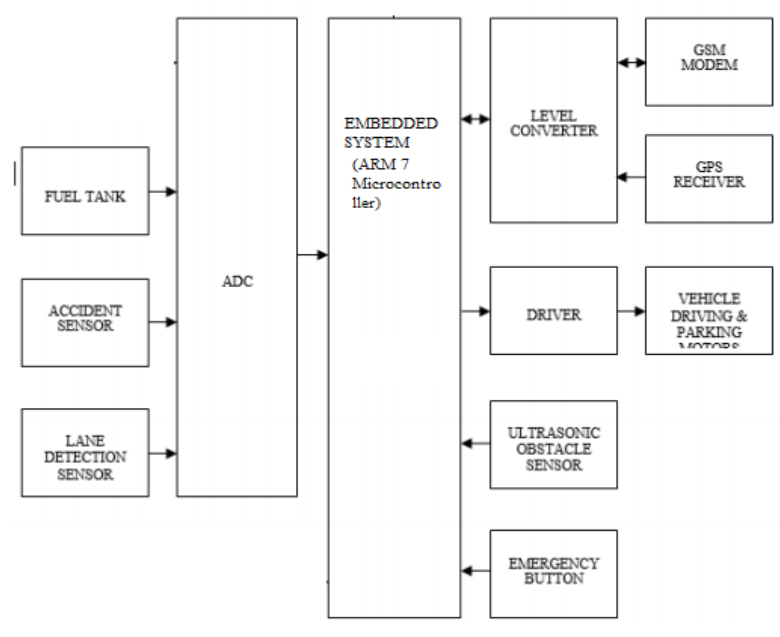

Fig 1. Proposed System

\subsection{Description of Each Block}

\subsubsection{Power Supply}

The performance of the whole circuit depends on the proper functioning of the power supply unit. The power supply pulls the required amount of electricity and converts the AC current to DC current. It also regulates the voltage to eliminate spikes and surges common in most electrical systems. The PSU used here provides $\mathrm{o} / \mathrm{p}$ voltage of 5volts, $1 \mathrm{amp}$. Not all power supplies, however, do an adequate voltage-regulation job, so the following essential components are used:

Transformer: A transformer transfers energy through electromagnetic induction. A varying current in the transformer's primary winding creates a varying magnetic flux in the core and a varying magnetic field impinging on the secondary winding. This varying magnetic field at the secondary induces a varying electromotive force (emf) or voltage in the secondary winding. Making use of Faraday's Law in conjunction with high magnetic permeability core properties, transformers are designed to efficiently change AC voltages from one voltage level to another within power networks. Transformer used here is a step down transformer used to step down from $230 \mathrm{v}$ to $+5 \mathrm{v}$. It also provides isolation from the mains.

Rectifier: A rectifier is an electrical device that converts alternating current (AC), which periodically reverses direction, to direct current (DC), which flows in only one direction. This design implements the full wave rectifier, using 1N4001 diodes. The bridge rectifier has advantage over the full wave rectifier - the need for the centre-tapped transformer is eliminated. The PIV is one half of the centretap circuit.

Filter: A filter circuit is a device which removes ac component of rectified output allowing only the dc component to reach the load. The filter used here is a simple capacitor of $100 \mu \mathrm{f} / 25 \mathrm{v}$.

Regulator: A voltage regulator is designed to automatically maintain a constant voltage level regardless of changes in load current. The regulator used in our project is IC7805, which is a three terminal voltage regulator. A heat sink is used, so that the heat produced by the regulator is dissipated over a larger area which holds the case temperature to a much lower value than would result without the heat sink. IC 7805 has an internal thermal overload protection and the internal short circuit current limiting device. 


\subsubsection{GPS Technology}

The Global Positioning System (GPS) is a spacebased satellite navigation system that provides location and time information in all weather conditions, anywhere on or near the Earth where there is an unobstructed line of sight to four or more GPS satellites. The system provides critical capabilities to military, civil and commercial users around the world. It is maintained by the United States government and is freely accessible to anyone with a GPS receiver. There are no subscription fees or setup charges to use GPS. A GPS receiver must be locked on to the signal of at least three satellites to calculate a 2D position (latitude and longitude) and track movement. With four or more satellites in view, the receiver can determine the user's 3D position (latitude, longitude and altitude). Once the user's position has been determined, the GPS unit can calculate other information, such as speed, bearing, track, trip distance, distance to destination, sunrise and sunset time and more. GPS receiver is used here to detect the location of the vehicle and provide information to responsible person through GSM technology.

\subsubsection{GSM Technology}

GSM/GPRS modem can be connected directly to your computers serial port for wireless GSM communications including sending and receiving text messages. It can also be connected to your remote RS232 serial equipment allowing you to "dial up" your system for remote management. This includes data loggers such as DataTaker and Pace Scientific. GPRS connectivity also allows you to integrate this modem in your applications for an "always on" remote management system. Available with a built-in TCP/IP stack and you can easily create software to interface to the modem over your standard internet connection.

Features:

- Tri-band GSM 850, 900, 1800 and

$1900 \mathrm{MHz}$

\section{- Supports TCP/IP}

- Accepts a standard SIM card

- Standard extended open AT commands

- Send/receive SMS text messages

- GPRS Class 10

- 5 to $12 \mathrm{~V}$ DC supply

- Dimensions: 115 x 65 x 27mm

\subsubsection{IR Sensing Circuit}

The IR sensing circuit can be used to detect an obstacle. IR Transmitter is an LED which emits infrared rays. IR Receiver is used to receive the IR rays. Both IR transmitter and receiver should be placed straight in line with each other. The transmitted signal is given to IR transmitter. Whenever the signal is high, the IR transmitter LED is conducting and it passes the IR rays to the receiver. The IR receiver is connected with comparator. The circuit is as shown in figure 2.

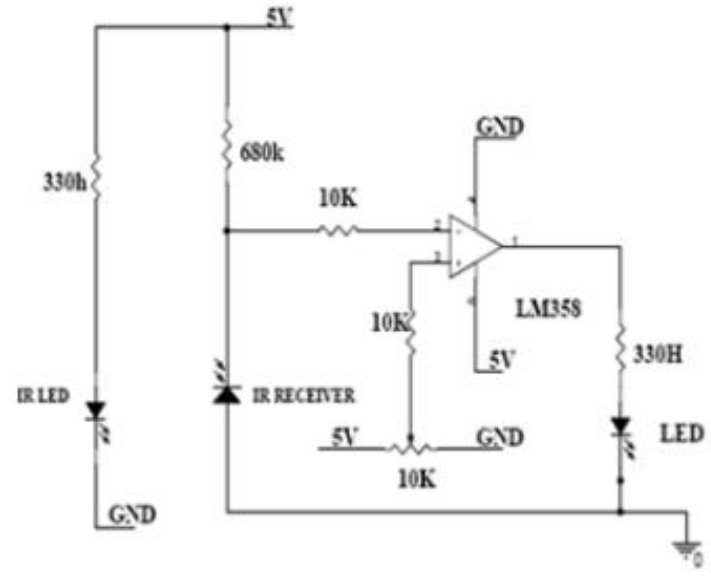

Fig 2. IC circuit

\subsubsection{Obstacle Sensing Module}

The obstacle sensing module is used to sense events such that accidents due to unwanted parking of the vehicles and collision with trees and other objects especially during the night time could be avoided. These obstacles could be detected using various methods like the ultrasonic sensors. Figure 3 shows the basic obstacle detection circuit.

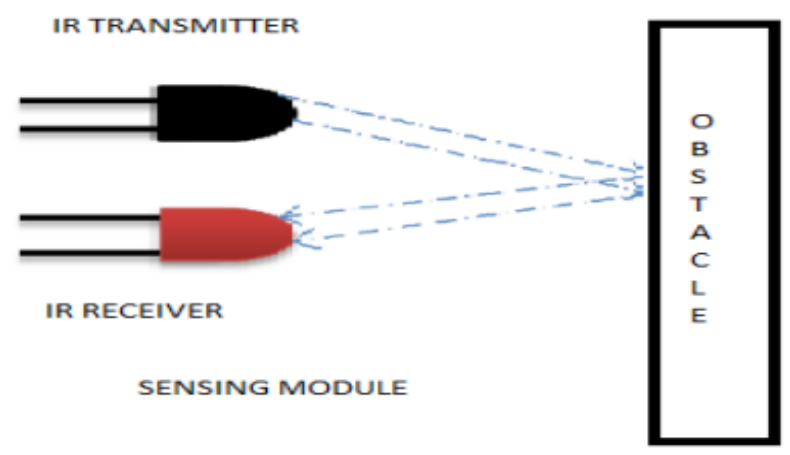

Fig 3. Obstacle detecting circuit

\subsubsection{ARM 7 Microcontroller}

ARM-7 microcontroller acquires and stores different parameter of vehicle. The main block of vehicle monitoring system is ARM-7 microcontroller which provides monitoring and controlling actions. It senses signals from input blocks and processes output blocks. The software program is stored in ARM-7 microcontroller on chip memory, according to which it provides the controlling actions. The on chip ADC converts these parameters into digital form and gives them to the ARM-7 microcontroller. The status of steering grip i.e. whether the steering is gripped properly or not is sensed by sensor, and it gives the corresponding signal to microcontroller.

With the help of different sensors various parameters are monitored. The LCD block is provided for visual display of the message. Also it continuously displays the measured parameters. The RTC provides real time clock depending on which the various events occur. Whenever accident takes place the accident interrupt block gives interrupt to the ARM7 microcontroller. Through serial communication block the system is interfaced with the PC. With this interfacing the stored data is transferred serially to PC, for the analysis purpose. 


\section{Features:}

- 16/32-bit ARM7TDMI-S microcontroller is a 64 or 144 pin package.Ashwini Y. Dakhole et al.., International Journal of Advance Research in Computer Science and Management Studies

- $16 \mathrm{~KB}$ on-chip Static RAM.

- $128 / 256$ KB on-chip Flash Program Memory. 128bit wide interface/accelerator enables high speed 60 $\mathrm{MHz}$ operation.

- In-System Programming (ISP) and In-Application Programming via on-chip boot-loader software, flash programming takes $1 \mathrm{~ms}$ per 512 byte line. Single sector or full chip erase takes $400 \mathrm{~ms}$.

- B Two 32-bit timers (with 4 capture and 4 compare channels), PWM unit (6 outputs), Real Time Clock and Watchdog.

- Multiple serial interfaces including two UARTs (16C550), Fast I2C (400 Kbits/s) and two SPIs60 $\mathrm{MHz}$ maximum $\mathrm{CPU}$ clock available from programmable on-chip Phase-Locked Loop.

- On-chip crystal oscillator with an operating range of $1 \mathrm{MHz}$ to $30 \mathrm{MHz}$

- $\quad$ Two low power modes Idle and Power-down.

\section{APPLICATIONS OF THE SYSTEM}

\subsection{For Personal Vehicle}

The proposed system can be used for a personal vehicle in which immediate help can be provided using SMS details to the vehicle fitted with this system. If any unfortunate accident occurs, the victimized car can be tracked using GPS technology.

\subsection{Insurance Companies}

Most of the times, a fake accident environment is set up to receive the claimed amount. So, insurance companies can implement this car system in the insured vehicle. The system would record every detail of the vehicle before as well as during the accident has occurred. The insurance company can easily analyze the data recorded and find out whether the accident was set up or it actually occurred. This will allow the insurance company to avoid giving falsely claimed money.

\subsection{Research and Development of Vehicle}

For testing the vehicle in $\mathrm{R}$ and $\mathrm{D}$, an engineer requires data about the vehicle at various speed and time. But this data is not available exactly as it is not possible to measure the data for every second and to measure the number of parameters at the same time. But if this proposed system is used, the data can be made available for each and every second with very high accuracy. With the help of any software (LABVIEW), the data can be plotted in graphical form of speed Vs time, and engine temperature Vs time.

\subsection{Military Applications}

Military vehicles carry ammunition from one place to another. Conspiracy hatchers, who know about this fact, may attack such vehicles, in order to either steal the arms or simply destroy them. In order to overcome the loss caused in this way, military vehicles can be fitted with this system. If the vehicle got attacked or damaged, an immediate SMS is sent to military base station and these ammunitions can be saved from reaching the wrong hands.

\subsection{Avoiding Fuel Theft}

This can be done by providing a threshold value to fuel engine which will not allow the amount of fuel to go below a certain level. If that happens then the owner will be informed through SMS

\section{CONCLUSION AND FUTURE WORK}

In this paper, we have proposed a novel method of vehicle tracking and locking systems used to track the theft vehicle by using GPS and GSM technology. When the theft identified, the responsible people send SMS to the micro controller, then issue the control signals to stop the engine motor. After that all the doors locked. To open the doors or to restart the engine authorized person needs to enter the passwords. In this method, easily track the vehicle place and doors locked.

In future, for Lane Tracking purpose a front camera can be attached to the vehicle. Long range IR sensors can also be used to avoid vehicle collision. Instead of a Microcontroller we can use a CPLD chip since the CPLD incorporates many more features than a Microcontroller. VLSI/VHDL can be used for CPLD programming. A finger print scanner can be incorporated on the steering wheel of the vehicle to allow only authorized user to access the vehicle. A system for detecting behavior of driver can also be implemented. A camera can be placed besides rear view mirror to check the behavior of the driver. If the driver is feeling drowsy or the driver is drunk then the speed of the vehicle can be reduced until it completely stops avoiding major mishaps.

\section{REFERENCES}

[1] S.Sonika, Dr.K.Sathiyasekar, S.Jaishree, (2014), Intelligent Accident Identification System using GPS, GSM modem, IJARCCE,Vol 3, Issue 2, pp 5487-5489

[2] Kai-Tai Song, Chih-Chieh Yang, of National Chiao Tung University, Taiwan, "Front Vehicle Tracking Using Scene Analysis", Proceedings of the IEEE International Conference on Mechatronics \& Automation 2005..

[3] Pau Muñoz-Benavent, Leopoldo Armesto, Vicent Girbés, J. Ernesto Solanes, Juan Dols, Adolfo Muñoz, and Josep Tornero, "Advanced Driving Assistance Systems for an Electric Vehicle" AUSMT, Vol 2, No 2, 2013

[4] Albert Alexe, R.Ezhilarasie, "Cloud Computing Based Vehicle Tracking Information Systems", ISSN: 2229 4333 ( Print) | ISSN: 0976 - 8491 (Online ) IJCST Vol. 2, Iss ue 1, March 2011

[5] M. Abhinaya et al, "Intelligent vehicle control using wireless embedded system in transportation system based on GSM and GPS technology", IJCSMC, Vol.3, Issue 9, September 2014, pg 244-258.

[6] Swami Supriya et al's "An intelligent vehicle control and monitoring using arm", International journal of engineering and innovative technology (IJEIT), Volume 2, Issue 4, October 2012

[7] Ashwini et al "Design of intelligent traffic control system using ARM", International Journal of Advance Research in Computer Science and Management Studies, Volume 1, Issue 6, November 2013

[8] Montaser et al "Intelligent anti-theft and tracking system for automobiles", International Journal of Machine Learning and Computing, Vol. 2, No. 1, February 2012 